\title{
Enhanced responsiveness and sensitivity to blue light by b/r-2 overexpression in Trichoderma atroviride
}

\author{
Correspondence \\ A. Herrera-Estrella \\ aherrera@ira.cinvestav.mx
}

Received 20 February 2007

Revised 2 July 2007

Accepted 5 July 2007

\section{E. U. Esquivel-Naranjo ${ }^{1,2}$ and A. Herrera-Estrella ${ }^{1,2}$}

\author{
${ }^{1}$ Departamento de Ingeniería Genética, Cinvestav Campus Guanajuato, Km 9.6 Libramiento \\ Norte Carretera Irapuato-León, A.P. 629, Irapuato 36500, Guanajuato, Mexico \\ ${ }^{2}$ Laboratorio Nacional de Genómica para la Biodiversidad, Cinvestav Campus Guanajuato, \\ Km 9.6 Libramiento Norte Carretera Irapuato-León, A.P. 629, Irapuato 36500, Guanajuato, Mexico
}

\begin{abstract}
Light is an environmental factor that regulates pivotal processes in living organisms, and appropriate perception is key to adaptation to the environment. Blue light activates asexual reproduction in Trichoderma atroviride through transcription factors BLR-1 and BLR-2 which regulate light-responsive genes. Here, we show that b/r-2 expression is a limiting factor for photoperception and photo-transduction. Overexpression of b/r-2 resulted in increased photoconidiation and stronger expression of light-induced genes. In contrast, overexpression of b/r-1 resulted in reduced photoconidiaton and weaker expression of light-induced genes. b/r-2 overexpression caused a marked reduction of growth when the fungus was grown under defined photoperiods, including a period of strong sensitivity to light, followed by a period of insensitivity. Long periods of incubation under this condition permitted recovery of a rhythmic growth similar to that of the wild-type. In addition, b/r-2 expression is apparently regulated at the post-transcriptional level through the BLR proteins and its expression level is BLR-1-dependent even in the dark. Finally, we demonstrated that b/r-2 overexpression caused higher sensitivity to blue light and we therefore propose that the preformation of BLR-1/BLR-2 complexes is key to adequate light perception in $T$. atroviride.
\end{abstract}

\section{INTRODUCTION}

Sunlight is a key environmental factor that determines the behaviour of living organisms. Thus proper perception of ambient light is essential for the adaptation of an organism to its environment. Among the diverse responses regulated by light, those commonly observed are associated with pivotal processes, such as growth, development, reproduction and metabolism (Linden et al., 1997; Franklin et al., 2005). In particular, the processes of blue-light perception, signal transduction and related responses have been extensively studied in bacteria, algae, plants and fungi.

In fungi, the best-studied responses to light are carotenogenesis, development, morphogenesis, tropism and resetting of the circadian clock (Idnurm et al., 2006; Linden et al., 1997; Liu et al., 2003; Lu et al., 2005; Silva et al., 2006). In Neurospora crassa, the proteins white collar 1 and 2 (WC-1 and WC-2) are essential for all its described light responses (Ballario et al., 1996; Linden \& Macino, 1997). Analysis of the deduced amino acid sequences of these proteins revealed that they are PAS (Per-Arnt-Sim)

Abbreviations: LOV domain, light, oxygen, voltage domain; LRE, lightresponse element; PAS domain, Per-Arnt-Sim domain; WC, white collar. domain-containing transcriptional factors with GATA type, zinc finger, DNA-binding domains. The WC-2 protein has only one PAS domain, whereas $\mathrm{WC}-1$ contains three PAS domains (A-C). WC-1 and WC-2 form complexes through the interaction of the PAS-C domain of WC-1 and the PAS domain of WC-2 (Cheng et al., 2002, 2003). These complexes bind to the light-response element (LRE) found in the promoters of light-regulated genes even in the dark (Froehlich et al., 2002; He \& Liu, 2005; Káldi et al., 2006). In the WC-1 protein, a specialized PAS domain essential for blue/UVA light perception, called LOV (light, oxygen, voltage), that binds FAD, similar to the first LOV domains described in plant phototropins, has been identified (Froehlich et al., 2002; He et al., 2002). WC- 1 acts as a photoreceptor that, in combination with WC-2, tunes light input directly by activating the expression of genes required for the different responses described in N. crassa (Froehlich et al., 2002; He et al., 2002). A group of early light-induced genes showing transient expression patterns, typical of adaptive responses, have been identified (Lewis et al., 2002). Among these genes, one encodes a second blue-light photoreceptor, VIVID, necessary for adaptation to constant illumination and to detect changes in light intensity (Schwerdtfeger \& 
Linden, 2003). Although other photoreceptors have been found in the N. crassa genome, it has not been possible to assign any function to them. Furthermore, genes homologous to $w c-1$ and $w c-2$ have been found in different fungi and they all encode proteins containing PAS domains, presumably used to form complexes that regulate light responses (Casas-Flores et al., 2004; Lu et al., 2005; Idnurm et al., 2006; Silva et al., 2006).

Trichoderma atroviride is a common soil fungus widely used as a biocontrol agent due to its capacity to parasitize phytopathogenic fungi of agricultural importance. It has been shown that its asexual reproduction (conidiation) is tightly regulated by light and nutrient availability (Horwitz et al., 1985). In T. atroviride, a pulse of blue/UVA light induces the synchronous production of conidia situated at the colony perimeter where the pulse was received. Two genes, $b l r-1$ and $b l r-2$, orthologous to $w c-1$ and $w c-2$ from $N$. crassa, have been identified and shown to be essential regulators of the expression of blue-light-responsive genes and photoconidiation (Casas-Flores et al., 2004; RosalesSaavedra et al., 2006). The BLR proteins contain PAS- and GATA-type DNA-binding domains that indicate their participation in a transcriptional factor complex, but seem to lack activation domains. PAS- and GATA-type binding domains have been found in WC-like proteins in other fungi, suggesting that they are sufficient for their role in light perception and subsequent transduction of the signal (Ambra et al., 2004; Lu et al., 2005; Idnurm et al., 2006; Silva et al., 2006). This is also supported by data demonstrating that a truncated form of WC-1, lacking its $\mathrm{N}$-terminal activation domain, is functional as a photoreceptor (Káldi et al., 2006). In addition, BLR-1 contains the LOV domain essential for binding FAD. All blue-lightregulated genes that are BLR-dependent strictly require both functional proteins, indicating that the BLR proteins act as a complex. By analogy with N. crassa, it is assumed that this complex regulates the expression of early blue-light-responsive genes through putative GATA type cis-elements present in the promoter region of blue-lightresponsive genes, similar to the LREs defined in $N$. crassa (Berrocal-Tito et al., 1999; Casas-Flores et al., 2004; Rosales-Saavedra et al., 2006). Interestingly, the BLR proteins participate in the light-dependent transcriptional activation and repression of early gene expression, a dual role previously not described in any other fungus (RosalesSaavedra et al., 2006). Additionally, a number of biochemical and molecular data have provided support for the existence of yet another blue-light perception pathway. It appears that activation of both blue-light perception pathways is necessary for the regulation of photoconidiation (Berrocal-Tito et al., 2000; Rocha-Ramírez et al., 2002; Casas-Flores et al., 2006). Furthermore, the identification of light-regulated genes allowed us to identify red-lightregulated genes (Rosales-Saavedra et al., 2006). These data clearly indicate that multiple light perception pathways are functional in T. atroviride, in agreement with the different photoreceptors recently identified in fungal genomes.
To better understand the role of blr-2 in the light perception and transduction pathways of $T$. atroviride, we analysed its transcriptional regulation and the effect of its overexpression on the light responses of this organism. We found that expression of $b l r-2$ is a limiting factor for light signal transduction and its overexpression leads to exacerbated responses to this stimulus. Furthermore, our results indicate that the expression of blr-2 is induced by light and autoregulated at the post-transcriptional level. Finally, blr-2-overexpressing strains showed higher sensitivity to light. In contrast, strains overexpressing $b l r-1$ were less responsive to light than the wild-type (WT). Our results indicate that BLR-2 plays key roles both in the perception and in the transduction of blue light in this fungus.

\section{METHODS}

Growth conditions, media and strains. Trichoderma atroviride WT strain IMI206040 and the corresponding $\Delta b l r-1$ and $\Delta b l r-2$ mutants were grown on PDA medium (Difco) at $27^{\circ} \mathrm{C}$. Conidiation of the $\Delta b l r-1$ and $\Delta b l r-2$ mutants was induced by mechanical injury to obtain inocula. Escherichia coli strain TOP10F' (Invitrogen) was used for plasmid DNA transformation.

To analyse growth in the dark, in photoperiods of $12 \mathrm{~h}$ dark/12 $\mathrm{h}$ light and in constant illumination with white light, the strains were grown on PDA in a controlled environment growth chamber operating at $27{ }^{\circ} \mathrm{C}$ (constant temperature) with a fluence of $230 \mu \mathrm{mol} \mathrm{m} \mathrm{m}^{-2} \mathrm{~s}^{-1}$, provided by cool-white fluorescent tubes. Petri plates $(15 \mathrm{~cm}$ diameter) containing PDA were inoculated in the centre with plugs of mycelia $(0.5 \mathrm{~cm}$ diam. $)$ and incubated for $72 \mathrm{~h}$ at $27{ }^{\circ} \mathrm{C}\left( \pm 1{ }^{\circ} \mathrm{C}\right)$. The radial growth rate of the strains was determined by measuring colony diameter every $12 \mathrm{~h}$. The experiment was carried out with six replicates for each strain.

Overexpression of blr genes in T. atroviride. Plasmid pCB1004, carrying the hygromycin B resistance marker (FGSC), was used as the backbone for the construction of a constitutive expression vector. A 550 bp fragment of the Aspergillus nidulans trpC terminator and a $750 \mathrm{bp}$ fragment containing the promoter of the Trichoderma reesei pkil gene were cloned by PCR using the primers: TtrpC-f ( $5^{\prime}$-GGTACCTAGTGATTTAATAGCTCC-3') and TtrpC-r (5'-GGTACCTGTGCATTCTGG-3') for the terminator, and Ppkil-f (5' ${ }^{\prime}$-CCGCGGCTCGAGATAACGGTG-3') and Ppkil-r (5'-CCGCGGTTAAGAGGGTTCTTC-3') for the promoter. KpnI (terminator) and SstII (promoter) sites in the primers (underlined) were added to facilitate cloning into plasmid pCB1004. Plasmids pHat $\alpha$ (Herrera-Estrella et al., 1990) and pZEGA (Mach et al., 1994) were used as templates for the amplification of the $\operatorname{trpC}$ terminator $(\mathrm{T} \operatorname{trp} C)$ and $p k i 1$ promoter (Ppki1), respectively, under the following PCR conditions: one initial cycle of $94{ }^{\circ} \mathrm{C}$ for $5 \mathrm{~min}, 30$ cycles of $94{ }^{\circ} \mathrm{C}$ for $30 \mathrm{~s}, 55{ }^{\circ} \mathrm{C}$ for $30 \mathrm{~s}$ and $68{ }^{\circ} \mathrm{C}$ for $45 \mathrm{~s}$, and one final cycle of $68{ }^{\circ} \mathrm{C}$ for $7 \mathrm{~min}$. The $\operatorname{trpC}$ terminator and pkil promoter were sequenced and cloned into the $K p n \mathrm{I}$ and SstII restriction sites of pCB1004, respectively, obtaining the expression vector pUE08. The correct orientation of the regulatory regions in pUE08 was confirmed by PCR using universal and reverse primers, in combination with the primers described above.

To overexpress the blr genes, the corresponding complete $b l r-2 \mathrm{cDNA}$ (1.6 kb) containing the UTR-5' and 3' ends (Casas-Flores et al., 2004) was cloned into the EcoRI site of pUE08, and the blr-1 ORF was cloned into the BamHI and HindIII sites of the same plasmid. The correct orientation of the $b l r-2$ cDNA was corroborated by restriction 
with different endonucleases. The plasmids were purified using the the Qiagen plasmid Midi Kit and used to transform T. atroviride protoplasts using the PEG- $\mathrm{CaCl}_{2}$ method described by Baek \& Kenerley (1998). All transformants were subjected to three rounds of monosporic culture. The identification and selection of the transformants was performed by PCR using primers pkil-f and $\operatorname{trp} C$-r.

Northern and Southern analysis. Genomic DNA was isolated following the procedure described by Raeder \& Broda (1985). Total RNA was isolated according to the protocol described by Jones et al. (1985). Southern and Northern blotting was performed using Hybond- $\mathrm{N}+$ membranes (Amersham), according to the manufacturer's recommendations. Filters were hybridized with probes labelled by random priming with $\left[\alpha-{ }^{32} \mathrm{P}\right] \mathrm{dCTP}$ and processed using standard procedures (Sambrook et al., 1989).

Analysis of light responses. T. atroviride cultures were grown in the dark for $48 \mathrm{~h}$ at $27^{\circ} \mathrm{C}$ on PDA plates and used as pre-inoculum. Mycelial plugs $(0.5 \mathrm{~cm}$ diam.) were taken from the colony growth front and placed on the centre of PDA plates with (for RNA extraction) or without (for photoconidiation analysis) a cellophane overlay. Cultures were allowed to grow for further $36 \mathrm{~h}$ under these conditions, and then photoinduced as described by Berrocal-Tito et al. (1999).

For analysis of the expression of light-responsive genes after a light pulse, colonies were exposed to $1200 \mu \mathrm{mol} \mathrm{m}{ }^{-2}$ (unless otherwise indicated) using a light source consisting of two cool-white fluorescent tubes filtered with LEE filter \#183 (fluence rate $5 \mu \mathrm{mol} \mathrm{m} \mathrm{m}^{-2}$ ) and placed back in the dark at $27^{\circ} \mathrm{C}$. Mycelial samples were subsequently collected at different times after exposure to light. At the indicated times, mycelia were scraped from the surface of the cellophane overlaying the PDA medium under low red safelight [LEE filter \#106 (fluence rate $0.1 \mu \mathrm{mol} \mathrm{m}^{-2} \mathrm{~s}^{-1}$ )] and immediately frozen in liquid nitrogen for RNA extraction.

For photoadaptation analysis, we followed the procedure described above, except that mycelia were kept under constant illumination.

Sensitivity to blue light was investigated following the procedure described above, except that colonies were exposed to a different fluence of blue light as indicated. Colonies were then placed back in the dark at $27{ }^{\circ} \mathrm{C}$ for $15 \mathrm{~min}$, and mycelia were collected and frozen in liquid nitrogen for RNA extraction.

Analysis of photoconidiation. Colonies were incubated for $48 \mathrm{~h}$ in the dark after photoinduction and conidia were collected in $8 \mathrm{ml}$ distilled water. Quantification of conidia was performed using an Axiostar Plus microscope (Zeiss) in a Newbauer chamber.

Analyses of blr gene expression by real-time PCR. Primers for the analysis of $b l r-1$ (blr1-f 5'-GAATGGCGGAGGGGGCCGAGT-3' and blr1-r $5^{\prime}$-CCGTTGCTGGGGATTGGATTTGGA-3') and $b l r-2$ (blr2-f $5^{\prime}$-GTAACCGCAGCCCTACCCTCATC-3' and blr2-r $5^{\prime}$ GCCCACCGCAACCCGCAGGC-3') gene expression by real-time PCR were designed. The primers gpd-f (5'-GGCTGCCGATGGTGAGCTCAAGGG-3') and gpd-r (5'-GAGGTCGAGGACACGGCGGGA-3') were designed to use the $g p d$ gene (encoding glyceraldehyde-3-phosphate dehydrogenase) as an internal loading and normalization control (Puyesky et al., 1997). Twenty micrograms of total RNA was obtained and, as described above, treated with amplification-grade RNase-free DNaseI (Invitrogen) and cleaned using RNeasy mini kit columns (Qiagen). cDNA synthesis was performed using $10 \mu \mathrm{g}$ RNA, a reverse primer mixture (gpd-r, blr1-r and blr2-r, 20 pmol each) and SuperScript RT III (Invitrogen), following the manufacturer's recommendations. After synthesis of the cDNA, the mixture was treated with $0.2 \mathrm{M} \mathrm{NaOH}$ for $15 \mathrm{~min}$ at
$37{ }^{\circ} \mathrm{C}$ to eliminate RNA and then neutralized with 0.7 M HEPES before purification of the cDNA by using QIAquick Spin columns (Qiagen). The optimal conditions and specificity for amplification of the three genes were determined by PCR using a Corbett Research thermocycler (version 2.2). Standard and relative efficiency curves were performed using the cDNA sample obtained from the WT strain grown in the dark to determine the dynamic range and to validate our reaction conditions for these genes. To this end, five serial dilutions $(1: 3)$ were made starting from $50 \mathrm{ng}$ to $0.61 \mathrm{ng} \mathrm{cDNA}$. The detection of blr-2 transcript in amounts below $0.1 \mathrm{ng}$ DNA was inconsistent. PCR amplifications were performed under the following conditions: 1 cycle of $95{ }^{\circ} \mathrm{C}$ for $10 \mathrm{~min}, 40$ cycles of $95{ }^{\circ} \mathrm{C}$ for $30 \mathrm{~s}, 64{ }^{\circ} \mathrm{C}$ for $30 \mathrm{~s}$ and $72{ }^{\circ} \mathrm{C}$ for $35 \mathrm{~s}$, using SYBR Green PCR Master Mix (Applied Biosystems). Five replicates were made for each experimental condition and all PCR reactions were performed in a $25 \mu \mathrm{l}$ final volume using a 7500 Real-Time PCR system (Applied Biosystems). The specificity of amplification was monitored and evaluated by the corresponding dissociation curves. The relative quantification of $b l r$ expression levels was performed with $6 \mathrm{ng}$ cDNA per reaction and their $\Delta \Delta C_{\mathrm{T}}$ was determined as a function of the calibrator condition (growth in the dark) to obtain comparative levels of relative expression for both blr genes after treatment with light, using the $2^{-\Delta \Delta C_{\mathrm{T}}}$ method (Livak \& Schmittgen, 2001). To compare the expression levels between $b l r-1$ and $b l r-2$, the $\Delta \Delta C_{\mathrm{T}}$ values were determined as a function of the $\Delta C_{\mathrm{T}}$ obtained in the dark for the $b l r-2$ gene.

\section{RESULTS}

\section{b/r-2 expression is induced by light}

The possible regulation of the blr genes by light was analysed by real-time PCR when Trichoderma was grown in the dark and exposed to a saturating pulse of blue light. We found increased levels of $b l r-2$ transcripts upon exposure of Trichoderma to the pulse of blue light, reaching its maximum level (27\% increase) compared to the dark control $30 \mathrm{~min}$ after the pulse. Then, the blr-2 transcript levels started to decrease, returning to the levels observed in the dark by $60 \mathrm{~min}$ (Fig. 1). In contrast, the expression of blr-1 decreased by $20 \%$ at $60 \mathrm{~min}$ after exposure to the light, coincident with the reduction of blr-2 expression. Upon comparison of the expression levels of $b l r-1$ and $b l r-$ 2, it was evident that the level of blr-1 transcripts was threefold higher than that detected for blr-2 (Fig. 1), suggesting that BLR-2 protein levels could be a limiting factor in blue light perception in T. atroviride. Thus, we decided to explore the effect of overexpression of blr-2 on the blue light response.

\section{Exacerbated photoconidiation by b/r-2 overexpression}

To test if blr-2 is a limiting factor for light input, we constructed the constitutive expression vector pUE08. The complete cDNA of blr-2 was cloned into plasmid pUE08. The resulting construct (pUE08-OEblr2) was used for transformation of $T$. atroviride. Out of 14 transformants analysed by PCR, only nine carried the transformation vector (data not shown). To demonstrate integration of the vector, we analysed the WT, the PCR-positive transformants, 


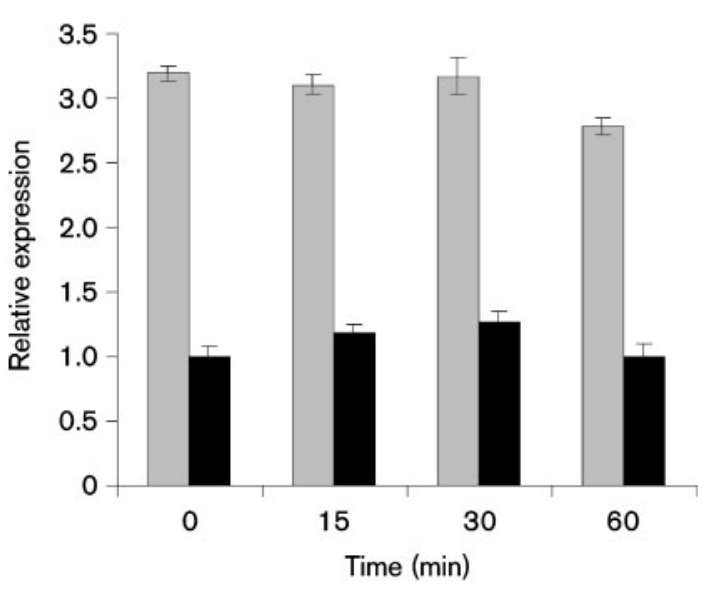

Fig. 1. Expression analysis of b/r-1 (grey bars) and b/r-2 (black bars) in the WT strain. Total RNA was isolated from the fungus grown in the dark (0) or collected at the indicated times after exposure to a pulse of blue light $\left(1200 \mu \mathrm{mol} \mathrm{m}{ }^{-2}\right)$, and subjected to real-time RT-PCR analysis. The expression levels of each gene were normalized using gpd as internal control, and the graphs show the mean expression levels relative to those found for blr-2 in the dark \pm SD.

named OEblr2 (over-expression of blr-2), and T-9, one of the PCR-negative putative transformants, by Southern blotting. The analysis, carried out using EcoRV-digested DNA, showed the expected banding pattern for the endogenous gene $(1.1$ and $5.6 \mathrm{~kb})$ in all strains, and the expected $0.6 \mathrm{~kb}$ band corresponding to the introduced copy of blr-2 was observed in all transformants, except T9. In addition, a $7.1 \mathrm{~kb}$ band was also observed in all transformants, except T-9 (Fig. 2a, b). The $7.1 \mathrm{~kb}$ band can only be explained if all integrations occurred at the same place. Indeed, homologous integration appears to be a highly frequent event in $T$. atroviride (unpublished data). Densitometric analysis of the hybridizing bands obtained indicated tandem integration of two copies of the vector in transformants OEblr2-1, -2, $-3,-4,-6,-8$, -12 and -14 , and three copies in OEblr2-13. To demonstrate the overexpression of blr-2 in the transformants, we carried out Northern blot analysis. All transformants carrying the construct, as confirmed previously by the Southern blot assays, showed high levels of $b l r-2$ transcripts, whereas in the WT strain and in strain T-9, used as an additional control, no detectable levels of blr-2 transcripts were observed (Fig. 2c).

Analyses of conidiation showed an exacerbated response to blue light in all blr-2-overexpressing strains, showing no signs of conidiation in the dark, indicating that this phenotype is totally dependent on light (Fig. 2d). In contrast, strain T-9 showed a phenotype identical to that of the non-transformed strain, indicating that the transformation process did not affect its capacity to respond to light. The production of conidia increased significantly in the $b l r-2$ overexpressers, with strain OEblr2-13 yielding the highest levels of conidia, producing 11.9-fold more conidia than the WT strain (Fig. 2e). Consistently, this strain was shown to carry three copies of the vector and to express the highest levels of the blr-2 transcript. These results are in agreement with the expression levels found for the blr genes and support our proposal that BLR-2 is a limiting factor in the phototransduction pathway of T. atroviride. Based on the results shown above, we selected the OEblr2-4 and OEblr2-13 strains, which represent all blr-2 overexpressers, for further analyses of light-regulated responses in $T$. atroviride.

\section{The BLR proteins modulate mycelial growth in the presence of light}

Previously, Casas-Flores et al. (2004) described a negative effect of light on mycelial growth of T. atroviride. Thus, we decided to investigate the effect of light on the growth of the blr-2-overexpressing strains compared to the WT strain, the transformation control strain T-9, and the gene replacement mutants $\Delta b l r-1$ and $\Delta b l r-2$. Under all conditions of growth analysed, we determined the radial growth every $12 \mathrm{~h}$ (radial growth rate). In the dark, the growth rate was similar among all strains, reaching a maximum after $24 \mathrm{~h}$. Moreover, a constant radial growth rate was maintained after $24 \mathrm{~h}$ of growth, without further apparent changes. Furthermore, the total radial growth was similar among all the strains (Fig. 3a, d). However, under constant illumination, the pattern of radial growth rate was modified in all the strains when compared to the corresponding dark controls. The $\Delta b l r$ strains reached a maximum growth rate by $24 \mathrm{~h}$, whereas the WT and T-9 strains reached their maximum between 36 and $48 \mathrm{~h}$, and OEblr2-4 and -13 reached it only at $48 \mathrm{~h}$ (Fig. $3 \mathrm{~b}$ ). The growth rate of all strains decreased gradually to a minimum by $72 \mathrm{~h}$. The growth pattern of the $\Delta b l r$ and OEblr2 strains showed an early maximum for the gene disruptants and a delayed maximum for the overexpressers, in contrast to the pattern observed for the WT strain. Under precise photoperiods, the growth rate was cyclic for the WT, T-9 and $\Delta b l r$ strains with faster growth rates in the dark and lower rates when exposed to light (Fig. 3c). Interestingly, OEblr2-4 and -13 showed an arrhythmic growth in the first four dark-light periods, developing an altered colony morphology, which recovered to a growth comparable to that of the rest of the strains by $60 \mathrm{~h}$ (Fig. 3c, e). Noticeably, the first light period provoked the strongest decrease in radial growth rate and the recovery was also slower in the next dark period. Interestingly, the blr-2overexpressing strains were apparently insensitive to a second light period as suggested by the constant radial growth rate observed during this period (Fig. 3c). This behaviour can be observed in Fig. 3(e), where the formation of two nearly continuous rings of conidia, corresponding to the first two light periods (central part with dense conidiation), was observed. In subsequent periods the spacing between the conidiation rings became 
(a)

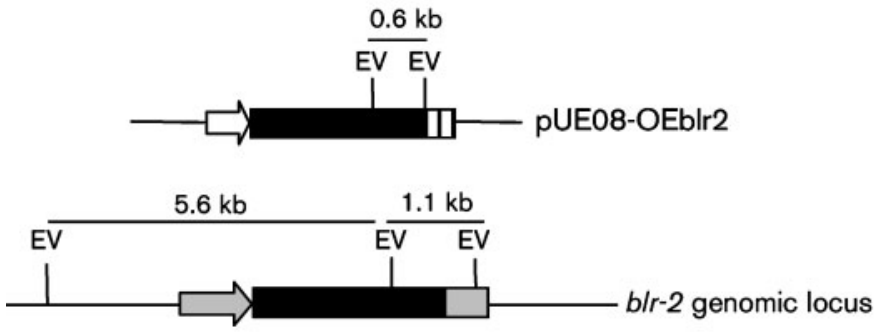

(c)

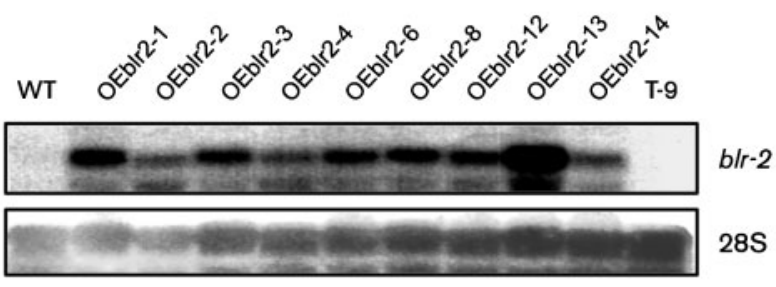

(b)

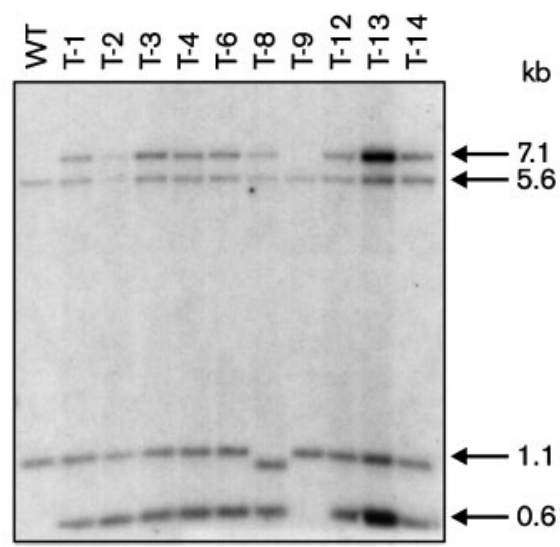

(d)

(e)

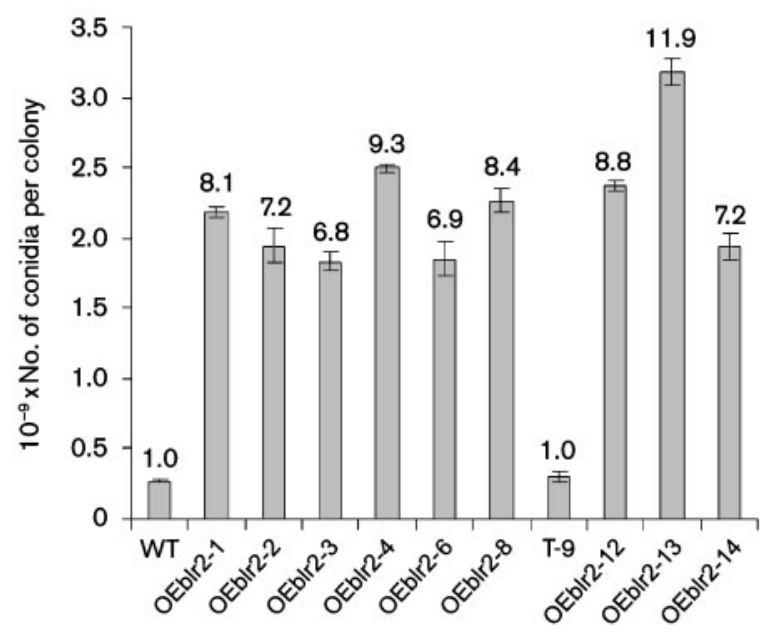

Fig. 2. Molecular and phenotypic analysis of b/r-2 overexpressers. (a) Schematic representation of vector pUE08-OEblr2 and the b/r-2 genomic locus. The number and position of the EcoRV (EV) sites are indicated. (b) Southern analysis. Twenty micrograms of total DNA of the indicated strains was digested with EcoRV and hybridized against ${ }^{32} \mathrm{P}$-labelled b/r-2 cDNA as probe. Molecular masses were estimated using a $1 \mathrm{~kb}$ ladder as marker. (c) Northern analysis. Twenty micrograms of total RNA isolated from mycelia grown in the dark were hybridized with ${ }^{32} \mathrm{P}$-labelled b/r-2 cDNA as probe. Hybridization with a $28 \mathrm{~S}$ rRNA probe was used as a loading control. (d) Photoconidiation. Colonies grown in the dark were exposed to a fluence of $1200 \mu \mathrm{mol} \mathrm{m}{ }^{-2}$, placed back in the dark for $48 \mathrm{~h}$ and photographed. (e) Quantification of conidia. The bars indicate the mean conidial yield per plate from three experiments; lines on bars indicate the mean value \pm SD and the numbers on the bars denote the increase in conidial yield relative to the WT strain.

similar among the different strains. The decrease in total radial growth could have been directly related to the arrhythmic growth provoked by the first two periods of exposure to light. This behaviour could not be associated with the higher photoconidiation of the blr-2 overexpressers, because the $\Delta b l r$ strains, which did not conidiate, had a rhythmic growth similar to that of the WT and T-9 strains (Fig. 3c, e). 
(a)

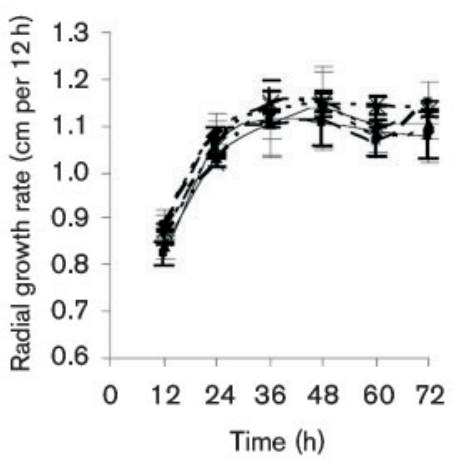

(d)

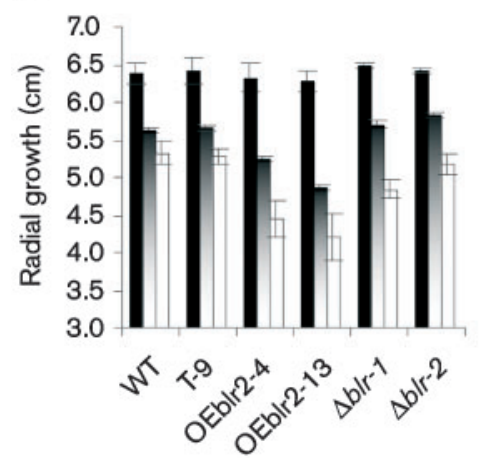

(b)

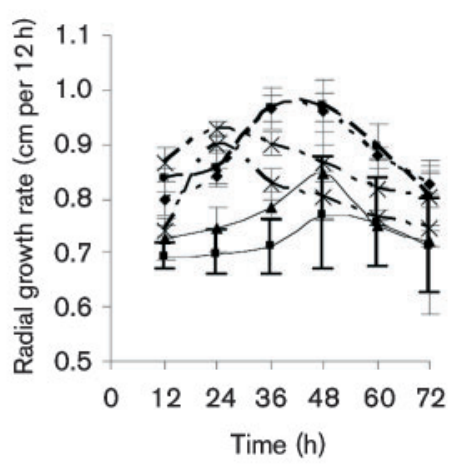

(e) (c)

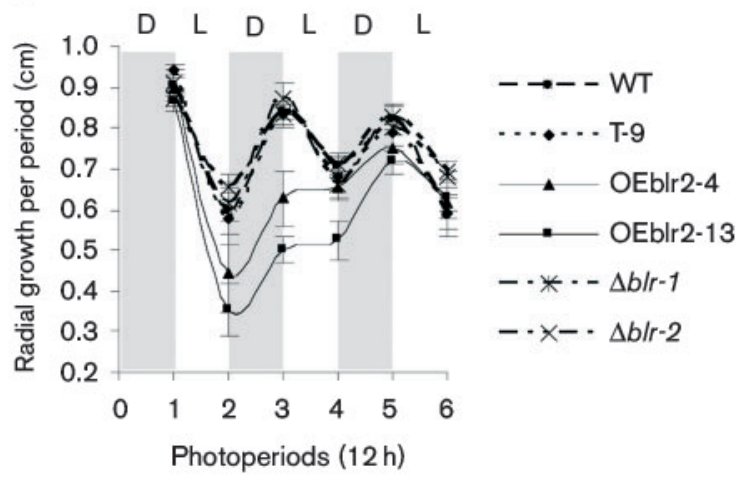

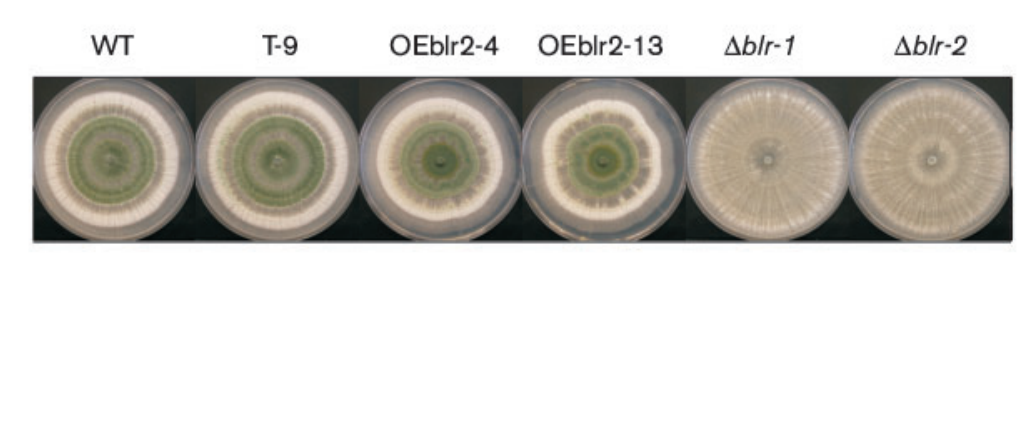

Fig. 3. Growth analyses of WT, OEblr2 and $\Delta$ b/r strains. As indicated, the strains were grown in the dark (a), under constant illumination with white light (b) and under light-dark photoperiods (12 h-12 h) (c), and their growth was measured as described in Methods. The graphs show the radial growth rate as measured every $12 \mathrm{~h}$ under the different growth conditions. (d) Total growth after $72 \mathrm{~h}$ for all strains employed under all conditions is plotted (white bars, light; shaded bars, light-dark; black bars, dark), and expressed as the mean of six experimental replicates \pm SD. (e) Photographs of the different strains after growth under light-dark photoperiods (12 h-12 h).

\section{Expression of blue-light-regulated genes takes place faster and reaches higher levels in b/r-2 overexpressers}

We have identified a set of novel light-responsive genes, named blue-light-upregulated $(b l u)$ and -downregulated (bld), including subsets of genes whose expression is BLRdependent and -independent, respectively (RosalesSaavedra et al., 2006). We analysed the expression of some of these genes in the blr-2-overexpressing and WT strains. The expression of all the light-inducible genes (blu-1, -4, $-7,-17$ and $p h r-1)$ was clearly higher in the OEblr2 strains (Fig. 4a). These genes showed different patterns of expression and could be divided into three well defined groups. In group 1, blu-7 showed rapid expression with a maximum at $15 \mathrm{~min}$, decreasing rapidly by $30 \mathrm{~min}$ after the light pulse, thus indicating a strong transient upregulation. In group 2, phr-1 and blu-17 showed an intermediate pattern of expression, reaching a maximum by 15-30 min and returning to basal levels of expression by $60 \mathrm{~min}$. In group 3, blu- 1 and $b l u-4$ showed a peak between 30 and 60 min after the light pulse, which decreased by $2 \mathrm{~h}$ after the stimulus (Fig. $4 \mathrm{a}-\mathrm{c}$ ). In all cases, the expression was faster and sustained for longer periods of time in the OEblr2-4 and OEblr2-13 strains, compared to the WT (Fig. 4a-c). Interestingly, the expression of blu-4, which was previously considered independent of or partially dependent on the BLR proteins (Rosales-Saavedra et al., 2006), was clearly affected by the overexpression of blr-2 (Fig. 4a).

Repression of bld-2 was apparently similar in both the WT and $b l r-2$-overexpressing strains. However, the dark-related recovery of expression was slower in the OEblr2-4 and OEblr2-13 strains, compared to the WT (Fig. 4a, d). These results provide further evidence of the role of the BLR proteins in the transcriptional activation and repression of blue-light-responsive genes.

\section{The expression of blr-2 is regulated through the BLR complex in both the dark and the light}

In $N$. crassa, the expression of the $w c$ genes is mainly regulated at the transcriptional level by means of the WC 
(a)
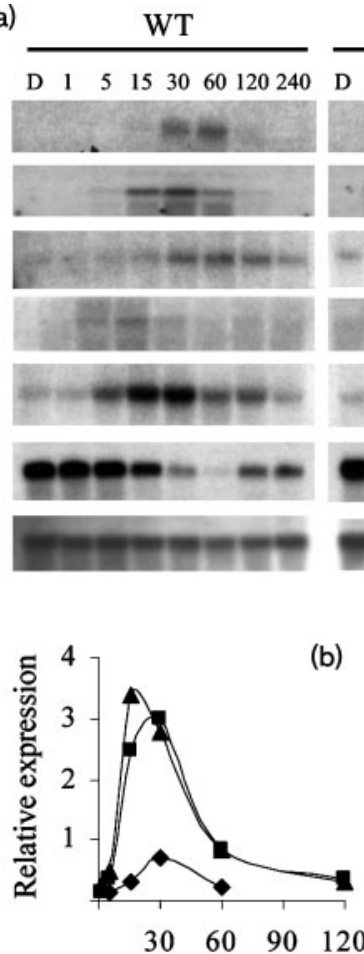
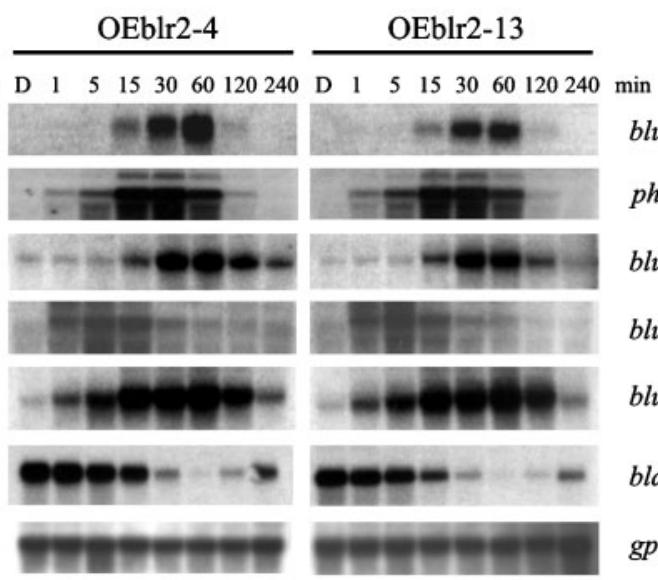

blu-1

phr-1

blu-4

blu-7

blu-17

bld-2

gpd

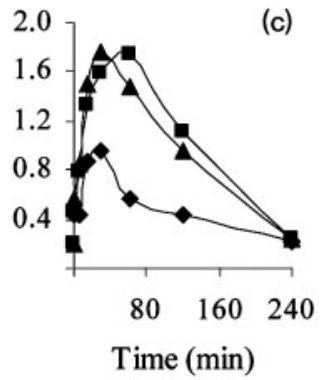

(d)

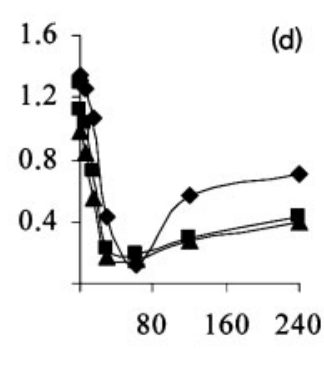

$80 \quad 160 \quad 240$
Fig. 4. Expression analysis of blue-light-regulated genes after a light pulse. (a) Colonies of the WT, OEblr2-4 and -13 strains were grown in the dark for $36 \mathrm{~h}$ and samples were collected (D) or further exposed to a pulse of blue light $\left(1200 \mu \mathrm{mol} \mathrm{m}{ }^{-2}\right)$ and collected at the times indicated after the pulse. Total RNA from the samples was isolated and subjected to Northern blot analysis. Blots were sequentially hybridized with ${ }^{32} \mathrm{P}$-labelled probes of the indicated genes. Hybridization with gpd was used as loading control. The time-course expression of phr-1 (b), blu-17 (c) and b/d-2 (d) was determined by densitometry analysis of scanned autoradiographs; values indicate expression levels relative to those of gpd, which was used as a loading control. $\$$ WT; 口, OEblr2-4; $\mathbf{\Delta}$, OEblr2-13. proteins. This WC protein dependence has been found both in the dark and after light induction (Ballario et al., 1996; Cheng et al., 2003; Káldi et al., 2006). Thus, we investigated, first, if $b l r-2$ overexpression had any effect on the expression of the blr genes and, second, if they were involved in regulating their own expression. In this regard, we found that $b l r-2$ expression levels in the dark were 12.4fold higher in the OEblr2-13 strain than in the WT strain, and up to 21.6-fold higher upon light induction (Fig. 5a), thus demonstrating that $b l r$-2-overexpressing strains express higher levels of $b l r-2$ than of $b l r-1$, an inverted situation to the behaviour found in the WT strain (Fig. 5a). Interestingly, the transcript levels of both $b l r$ genes increased similarly in response to blue light, showing a $75 \%$ increase for both $b l r-1$ and $b l r-2$ in the OEblr2-13 strain, and the transcript level of $b l r-1$ fluctuated over time (Fig. 5a). Furthermore, light-induced accumulation of blr2 transcripts in the OEblr2-13 strain was faster and stronger, reaching its maximum level $15 \mathrm{~min}$ after a light pulse. This change in $b l r-2$ expression is similar to the expression pattern found in the blue-light-upregulated genes (Fig. 4a), indicating that it regulates its own expression (Fig. 5a). Noticeably, the $75 \%$ increase in the $b l r-2$ transcript levels upon light induction in the $b l r-2$ overexpresser represents a 9.2-fold increase compared to the level found in the WT strain. Northern blot analysis of a $T$. atroviride transformant carrying a transcriptional fusion of the gene encoding the green fluorescent protein $(g f p)$ with the $p k i-1$ promoter indicated that there were no significant changes in $g f p$ expression in response to light (data not shown). Furthermore, conidiation and the expression of blu genes induced by light in that strain were similar to those of the WT. Given the fact that the overexpression of $b l r-2$ was achieved using a constitutive promoter, these data suggest autoregulation at the posttranscriptional level. In the dark, the levels of $b l r-1$ expression were not altered in the $b l r-2$ overexpresser (Fig. 5a), directly linking the observed phenotypes to $b l r-2$ overexpression. Additionally, we analysed the expression of $b l r-2$ in the $\Delta b l r$ 1 strain to investigate the dependence of $b l r-2$ expression on BLR-1. We found approximately 3.5-fold lower levels of blr-2 expression in the mutant compared to the expression levels in the WT strain, even in dark (Fig. 5b), indicating that BLR1 is necessary to maintain the expression of $b l r-2$ in the absence of light. Furthermore, the light induction of the steady-state levels of $b l r-2$ transcripts in the $\Delta b l r-1$ strain was not observed. Thus, our data suggest that the BLR complex regulates the induction of blr-2 transcripts occasioned by light at the post-transcriptional level.

\section{Expression of b/r genes is altered by b/r-2 overexpression under constant illumination}

Blue-light-regulated genes are subjected to photoadaptation in T. atroviride, as occurs in other organisms (RosalesSaavedra et al., 2006). Therefore, we analysed the expression of the blr genes under constant illumination. The expression of $b l r-2$ in the WT strain under constant blue light was upregulated by $40 \%$ and showed an expression pattern identical to the induction provoked by 


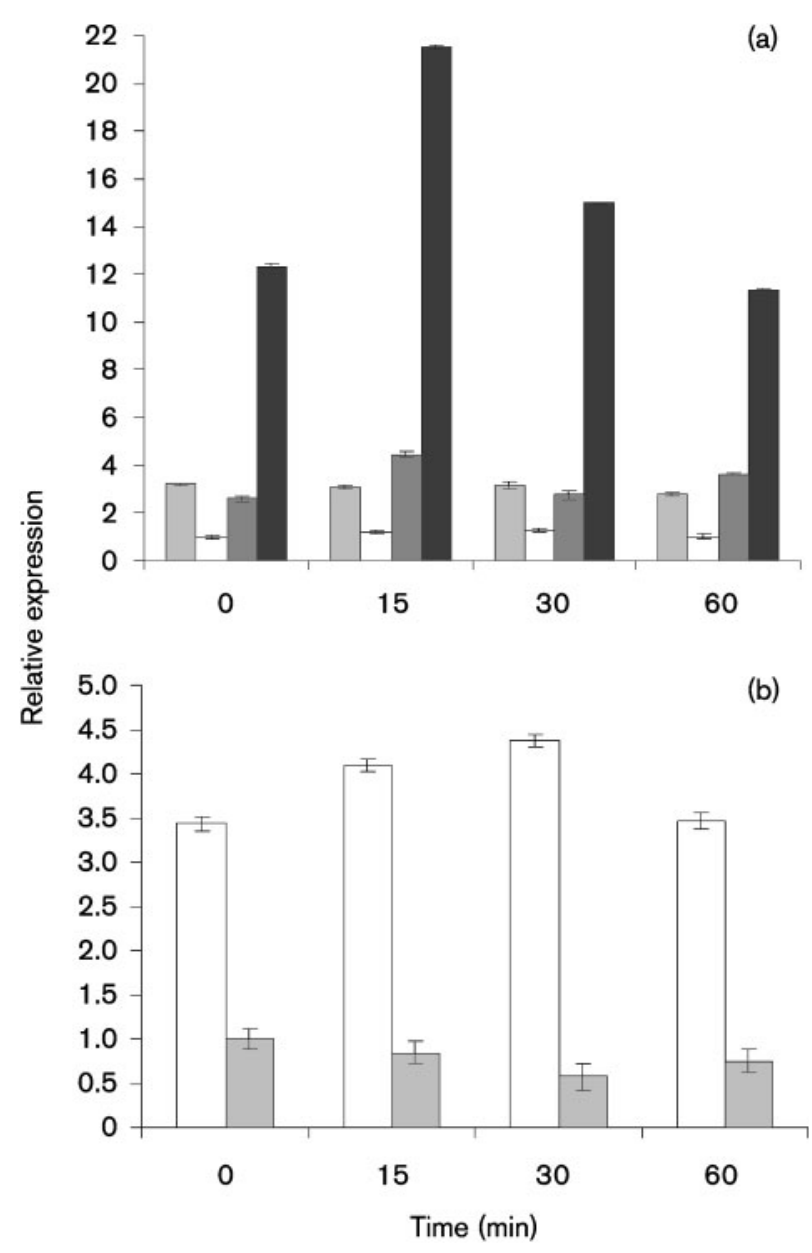

Fig. 5. Expression of b/r genes after a light pulse in the WT, OEblr2-13 and $\Delta b / r-1$ strains. (a) Expression of $b / r$ genes in OEblr2-13 and WT strains. Light grey bars, WT (b/r-1); white bars, WT (b/r-2); mid-grey bars, OEblr2-13 (b/r-1); dark grey bars, OEblr2-13 (b/r-2). (b) Expression of blr-2 in the WT (white bars) and $\Delta b / r-1$ (grey bars) strains. cDNA was synthesized from total RNA isolated from the indicated strains grown in the dark (0) and at the times indicated after exposure to a blue light pulse $\left(1200 \mu \mathrm{mol} \mathrm{m}{ }^{-2}\right)$. The results were normalized using gpd as an internal control and the -fold change after a light pulse is expressed relative to mRNA levels of b/r-2 found in the dark for (a) WT and (b) $\Delta b / r-1$ strains. The graphs show the mean expression levels $\pm \mathrm{SD}$.

a light pulse, with transcript levels decreasing to those found in the dark (Fig. 6a) after $60 \mathrm{~min}$. However, the expression pattern of blr-2 was modified in the OEblr2-13 strain, losing the typical transitory expression found in the WT strain (compare Fig. 6a and Fig. 6b). Light induction was only detected after 30-60 min of continuous exposure to light, showing a similar increase in transcript levels of $40 \%$ in both blr genes, but no decrease was observed in the time frame analysed (Fig. 6b, c). Furthermore, expression of blr-1 under constant illumination was different in both strains. In the WT strain, light had a modest negative effect on blr-1 expression ( $25 \%$ decrease) that apparently coincided with a decrease in blr-2 transcript levels (data not shown), whereas in the OEblr2-13 strain light stimulated blr-1 expression (Fig. 6c). These results indicate altered light regulation of the blr genes under constant illumination occasioned by the overexpression of $b l r-2$.

We analysed the expression of light-regulated genes to determine if the changes in the expression patterns of the blr genes were associated with a general alteration of photoadaptation caused by blr-2 overexpression. Under the same conditions, we found that the expression pattern of light-regulated genes was similar between the WT and OEblr2-13 strains. Although it appeared that the messengers decreased more slowly in OEblr2-13 than in the WT strain, this may be only an effect of the higher levels of transcripts accumulated in this strain (Fig. 6d-f). In addition, repression of the bld-2 gene was also comparable in both strains, even though recovery of the transcript levels observed in the dark was not evident in the time frame analysed here, considering that its expression starts increasing only after $6 \mathrm{~h}$ of growth under these conditions (data not shown). These results suggest that blr-2 overexpression does not alter the photoadaptative response of the light-regulated genes of Trichoderma and that a different mechanism regulates the temporary expression of the blr genes under constant illumination.

\section{A high level of b/r-2 expression enhances sensitivity to blue light in $T$. atroviride}

Based on the possibility that the BLR proteins act as a complex, we rationalized that the formation of such complexes in the dark should be important for appropriate light perception. Therefore, blr-2 overexpression should result in changes in the sensitivity to blue light. In this context, we decided to investigate if the blr-2 overexpressers were altered in their photosensitivity. The WT strain, and the OEblr2-4 and -13 strains were exposed to different fluences of blue light. As shown in Fig. 7(a), both transformants showed a conidiation ring when exposed to $225 \mu \mathrm{mol}$ blue light $\mathrm{m}^{-2}$, comparable to the ring observed in the WT strain when exposed to $1200 \mu \mathrm{mol} \mathrm{m}{ }^{-2}$ using the same light source. Statistical analysis of the fluence response allowed us to estimate a saturation dose of 2400 and $1200 \mu \mathrm{mol} \mathrm{m}{ }^{-2}$ for the WT and OEblr2 strains, respectively (Fig. 7b). The half-saturating doses for photoconidiation were $1859 \mu \mathrm{mol} \mathrm{m}^{-2}$ for the WT strain, and 612 and $560 \mu \mathrm{mol} \mathrm{m}{ }^{-2}$ for OEblr2-4 and -13 , respectively, indicating a greater than threefold higher sensitivity of both transformants than that detected in the WT strain (Fig. 7b). The difference between the two transformants was statistically not significant, indicating that the BLR-2 levels were saturating, even in the OEblr2-4 strain, which had lower $b l r-2$ expression. Additionally, the yield of conidia was evidently higher in the blr-2-overexpressing strains than that in the WT strain (Fig. 7a, b). 


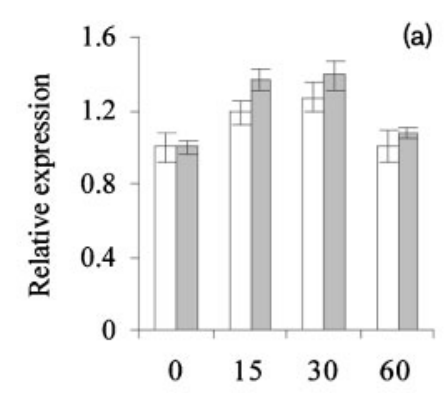

(d)

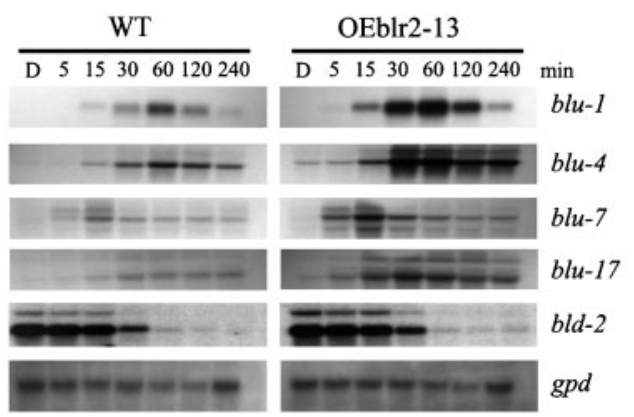

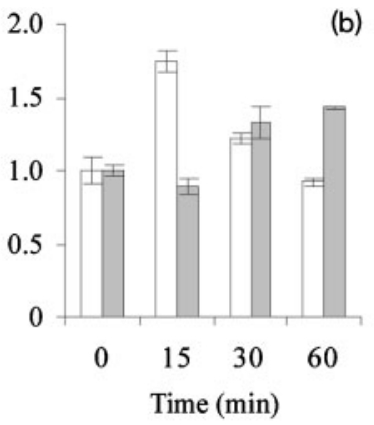

(b)
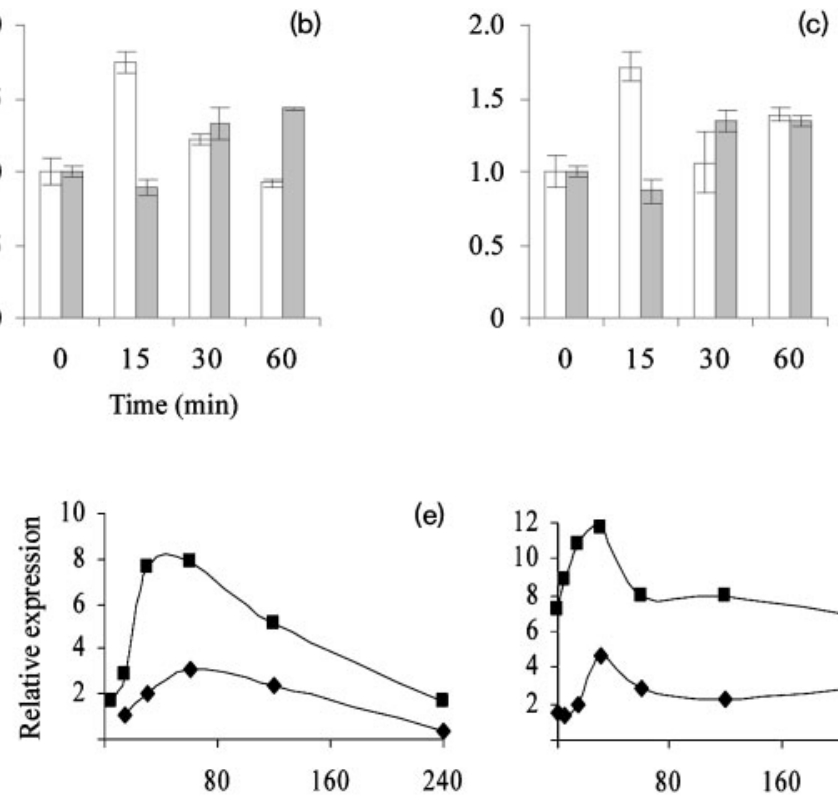

(c)

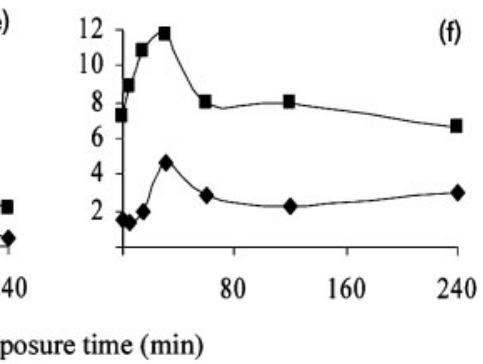

Fig. 6. Expression analysis of $b / r$ and blue-light-regulated genes under constant illumination. (a-c) Expression of $b / r-1$ and $b / r-$ 2 genes under constant illumination with blue light. Comparative expression levels of the b/r-2 transcript after a light pulse (white bars) and under constant illumination (grey bars) in the WT (a) and OEblr2-13 (b) strains. The b/r-2 expression level in the dark was normalized to 1 for each experimental condition. (c) b/r-1 expression levels in the OEblr2-13 strain under constant illumination and after a light pulse at the indicated times. The level of b/r-1 expression in the dark was normalized to 1 . All data was normalized using gpd as internal control. (d) Expression analysis of blue-light-regulated genes. Total RNA (20 $\mu \mathrm{g})$ isolated from mycelia grown in the dark (D) or exposed to continuous blue light of the WT and OEblr2-13 strains was collected at the indicated times and subjected to Northern blot analysis. Blots were sequentially hybridized with ${ }^{32} \mathrm{P}$-labelled probes of the cDNA clones for the indicated genes. Hybridization with gpd probe was used as a loading control. The time-course expression profile was determined by densitometry analysis of the scanned autoradiographs for blu-1 (e) and blu-17 (f) using the loading control (gpd) for normalization. WT; $\mathbf{0}$, OEblr2-13.

Similar results were obtained for the expression of bluelight-regulated genes. The overexpression of $b l r-2$ caused a higher accumulation of mRNA for the light-responsive genes, at the same light fluence, than that observed in the WT strain (compare OEblr2 and WT in Fig. 7c). Furthermore, blu-1 and phr-1 reached their maximum expression, in the OEblr2 strains, with a fluence of $600 \mu \mathrm{mol} \mathrm{m}{ }^{-2}$, whereas in the WT strain their expression was still increasing when $1200 \mu \mathrm{mol} \mathrm{m}{ }^{-2}$ was applied. In the case of blu-7 and $b l u-17$, the response was saturated at $300 \mu \mathrm{mol} \mathrm{m}{ }^{-2}$ in the OEblr2 strains, whereas saturation in the WT strain was reached using $600 \mu \mathrm{mol} \mathrm{m}{ }^{-2}$. The expression of $b l u-4$ did not appear to reach saturation even when exposed to the maximum fluence used in this analysis (Fig. 7c). Taken together, these results show that the higher photosensitivity and early saturation with light are directly associated with the overexpression of blr-2.

\section{Effect of blr-1 overexpression in photoconidiation and gene expression}

To provide further support to our hypothesis of blr-2 expression being a limiting factor in light signal transduction, we cloned the complete cDNA of blr-1 into plasmid pUE08. The resulting construct (pUE08-OEblr1) was used for transformation of $T$. atroviride. Out of 40 patitive transformants analysed by PCR, only eight carried the transformation vector and were confirmed by Southern analysis (data not shown). Northern analysis of the transformants carrying the construct showed, in all cases, high levels of blr-1 transcript even in the dark, whereas in the WT and OEblr213 strains no detectable levels of $b l r-1$ transcript could be observed (Fig. 8a). Unexpectedly, overexpression of blr-1 caused a strong decrease in light-induced conidiation compared to the WT and OEblr2 strains (Fig. 8b). Additionally, photoconidiation of the blr-1-overexpressing strains induced by different doses of light was always lower than for the WT and OEblr2-13 strains. However, conidiation induced by mycelial injury was similar among all strains (data not shown), indicating a light-specific alteration.

Because of the negative impact of $b l r-1$ overexpression on photoconidiation, we analysed the effect of blr-1 overexpression on light-induced gene expression. Accordingly, analysis of the expression of blue-light-regulated genes after a light pulse in a selected strain overexpressing blr-1 
(a)

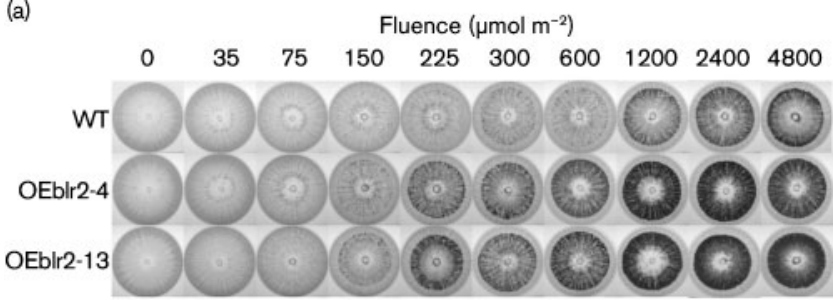

(b)

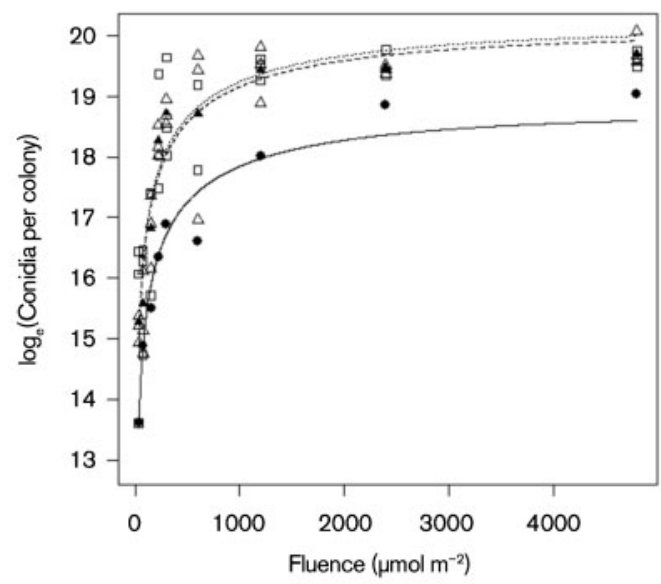

(c)

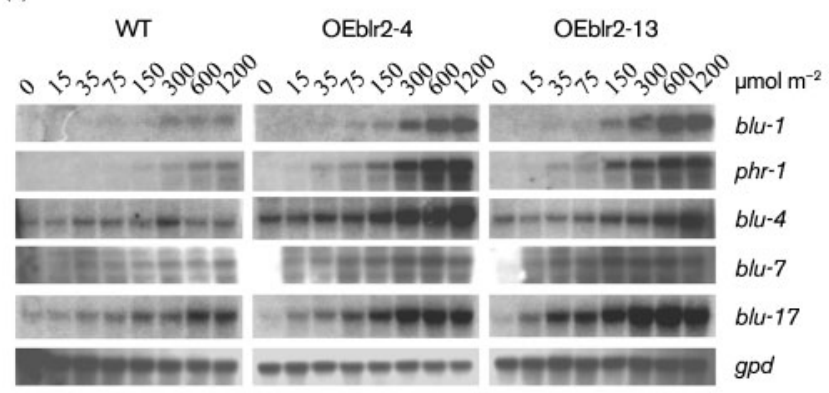

Fig. 7. Photoconidiation and light-regulated gene expression under different light doses. ( $a, b)$ Photoconidiation. Colonies grown in the dark were exposed to the indicated fluences of blue light, incubated for a further $48 \mathrm{~h}$ in the dark and photographed (a), or the conidia were quantified (b). Circles, WT; squares, OEblr2-4; triangles, OEblr2-13. Open symbols and filled symbols represent raw data and mean values for the three replicates of the experiment, respectively. (c) Expression of blue-light-regulated genes. Northern blot analysis of $20 \mu \mathrm{g}$ total RNA isolated from mycelia of WT, OEblr2-4 and -13 strains grown in the dark (0) or exposed to a pulse of blue light of the indicated fluence. RNA was extracted $15 \mathrm{~min}$ after the pulse of light. Blots were sequentially hybridized with ${ }^{32} \mathrm{P}$-labelled probes of the cDNA clones of the indicated genes. Hybridization with gpd probe was used as loading control.

showed lower levels of blu-1 and blu-17 transcripts compared to that observed for the WT and OEblr2-13 strains under the same conditions. The negative effect of $b l r-1$ overexpression on the regulation of blu-1 and blu-17 was clearly different, with $b l u-1$ showing a much lower level of induction. Downregulation of bld-2 was less drastic
(Fig. 8c). These results suggest that the regulation and levels of the BLR proteins are very important for appropriate induction of gene expression.

\section{DISCUSSION}

Homologues of the WC proteins have been found in other Ascomycetes, as well as in the Basidiomycetes and Zygomycetes (Ambra et al., 2004; Casas-Flores et al., 2004; Idnurm et al., 2006; Lu et al., 2005; Silva et al., 2006). In $T$. atroviride, $b l r-1$ and $b l r-2$ encode essential proteins for photoconidiation and regulation of a set of blue-lightresponsive genes (Casas-Flores et al., 2004; RosalesSaavedra et al., 2006). The expression of these genes is apparently very low, similar to the level found for the Cryptococcus neoformans cwc genes (Lu et al., 2005). The blr-2 transcript levels were lower than those detected for $b l r-1$. T. atroviride strains overexpressing $b l r-2$ had a higher yield of conidia in response to blue light. Our results are consistent with the hypothesis that $b l r-2$ expression is a limiting factor in the transduction of the light signal. In $N$. crassa, WC-1 is limiting, compared to WC-2, and its overexpression enhanced the expression levels of bluelight-regulated genes and the rhythmic expression of $\mathrm{frq}$ (Cheng et al., 2001; Franchi et al., 2005). Consistent with the higher levels of WC-2 compared to WC-1, overexpression of $w c-2$ did not cause any obvious photosensorial alteration in $N$. crassa.

Exposure of $T$. atroviride to continuous white light results in a reduction in growth, even in blr deletion mutants. This effect was intensified when only the blue or red regions of the spectrum were applied (Casas-Flores et al., 2004). Additionally, growth of a $T$. reesei strain with a mutation in envoy, encoding a putative secondary light receptor (ENVOY), was strongly affected by white light (Schmoll et al., 2005). In Tuber borchii and N. crassa, light also affects radial growth during light-dark periods which is partially modulated through WC-1 (Ambra et al., 2004). We did not detect significant differences among the different strains analysed when grown in the dark. However, notable changes were observed during growth under constant illumination and during photoperiods. In all cases, light had a negative effect on growth, being stronger in the blr-2 overexpressers. Under constant illumination, we found different growth patterns with consistent radial growth rate profiles among the phenotypes analysed (WT, OEblr2 and $\Delta b l r)$, therefore suggesting that the BLR proteins modulate T. atroviride growth as proposed by Casas-Flores et al. (2004). Under defined photoperiods, we found a rhythmic growth rate in the WT strain and the $\Delta b l r$ mutants. Interestingly, the $b l r-2$ overexpressers showed a temporary arrhythmic growth in the first period of exposure to light. This growth alteration was slightly stronger in OEblr2-13 than in OEblr2-4, directly correlating the observed phenotype with the level of expression of $b l r-2$. Furthermore, there was a clear state of insensitivity in the second light period, suggesting an alteration of 

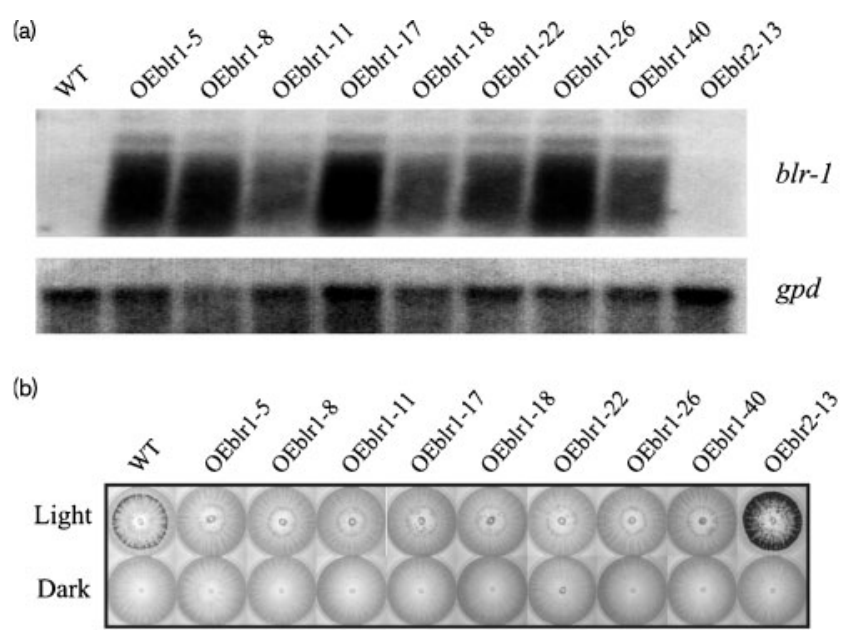

(c)

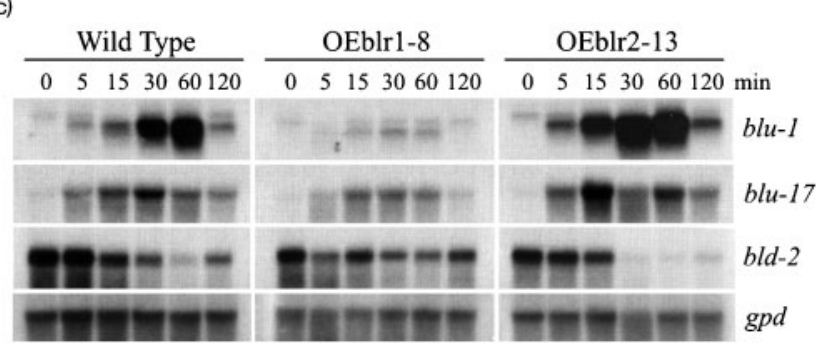

Fig. 8. Molecular and phenotypic analysis of $b / r-1$ overexpressers. (a) Northern blot analysis. Total RNA $(20 \mu \mathrm{g})$ isolated from mycelia grown in the dark was hybridized with ${ }^{32} \mathrm{P}$-labelled probe of the b/r-1 cDNA. Hybridization with gpd probe was used as loading control. (b) Photoconidiation. Colonies grown in the dark were exposed to $1200 \mu \mathrm{mol} \mathrm{m}{ }^{-2}$, placed back in the dark for $48 \mathrm{~h}$ and photographed. (c) Expression analysis of light-regulated genes for the WT, OEblr1-8 and OEblr2-13 strains after a light pulse as described in the legend to Fig. 4(a).

photoadaptation in the blr-2-overexpressing strains. However, we did not find significant changes in the expression pattern of light-regulated genes under continuous light exposure, indicating that various mechanisms may be operating in photoadaptation of $T$. atroviride. Although the envoy mutant is defective in growth under constant illumination, and the corresponding gene is clearly light-regulated (Schmoll et al., 2005) through the action of BLR proteins (unpublished data), we did not observe such strong inhibition in the $\Delta b l r$ mutants, indicating that, at least in part, the BLR proteins are negative modulators of mycelial growth and that ENVOY may participate in a negative feedback loop that desensitizes the light input through the BLR complex, as indicated in Fig. 9(a). Furthermore, the fact that this growth disturbance could be observed even in the $\Delta b l r$ strains strongly suggests the participation of an alternate light perception system, which at some point must establish cross-talk with the BLR-dependent system (Fig. 9a). Noticeably, this growth disturbance is temporary and the recovery after the fourth photoperiod suggests the (a)

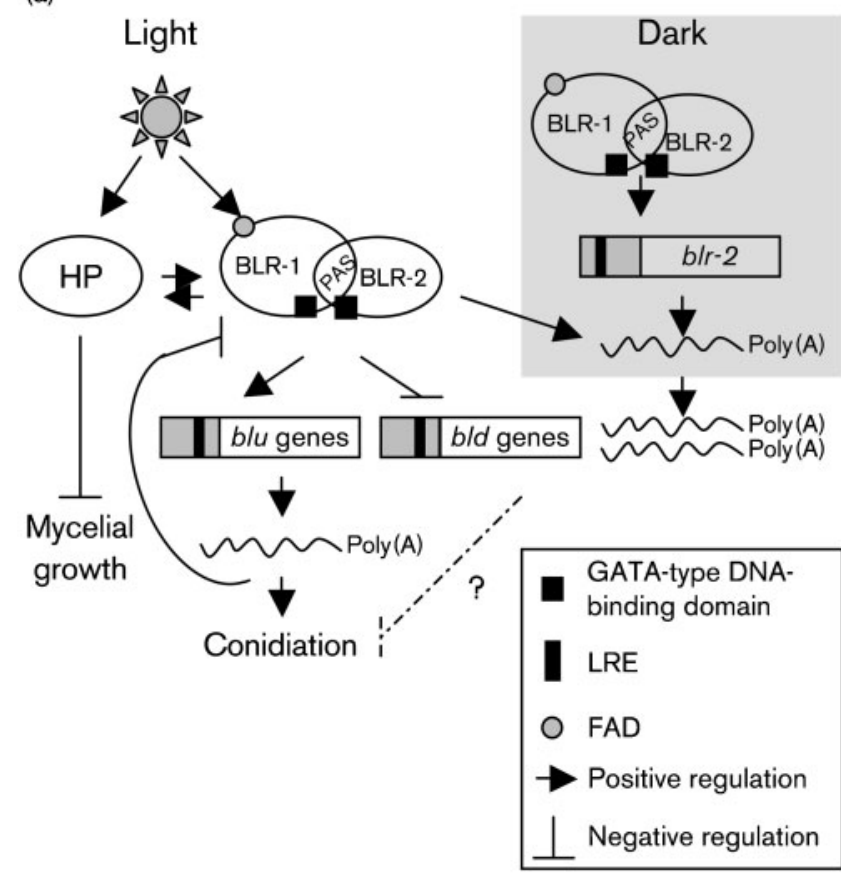

(b)

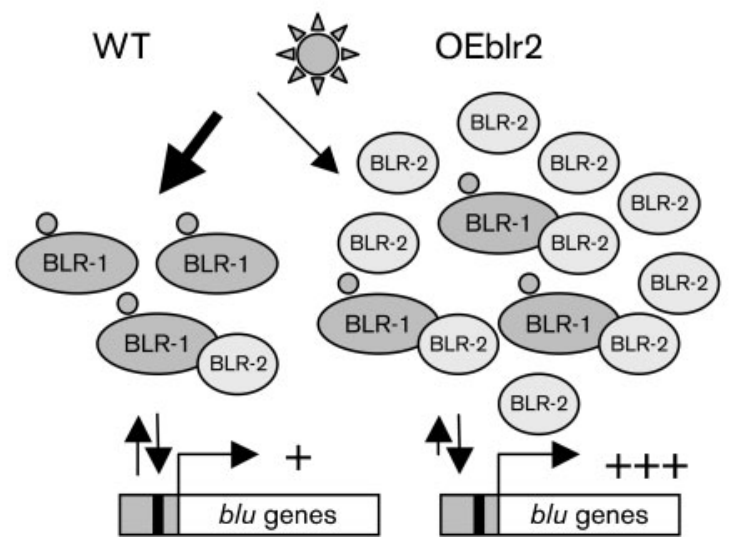

Fig. 9. Model for light regulation of gene expression through the BLR complex in T. atroviride. (a) Light is sensed through the LOV domain of BLR-1, activating the BLR complex, which in turn regulates the expression of light-responsive genes (b/u and b/d), through LREs present in their promoter regions and subsequent photoconidiation. The BLR complex regulates b/r-2 expression at the transcriptional level in the dark (grey area), and at the posttranscriptional level after light induction. The expression of lightregulated genes is transient under constant illumination, a process that is putatively regulated through a negative feedback loop where ENVOY could be part of that circuit. White light reduces the growth of $T$. atroviride through a hypothetical photoreceptor (HP) that interacts with the light input through BLR proteins. (b) BLR-2 is a limiting factor in light perception in $T$. atroviride (left panel), and its overexpression saturates the blue light perception system through the formation of a BLR complex, leading to the generation of more photoreceptor complexes (right panel).

existence of interrelated feedback loops among different light perception systems. 
A set of blue-light-regulated genes whose expression was both dependent and independent of BLR proteins has been identified in T. atroviride (Rosales-Saavedra et al., 2006). All genes analysed showed an earlier and stronger response to a light pulse in OEblr2 strains, compared to the WT strain. These results were consistent with the higher level of photoconidiation observed, directly associated with blr-2 overexpression. Overall, our results clearly indicate that $b l r-2$ expression is a limiting factor in light signal transduction as demonstrated by the lower transcript levels detected compared to those detected for $b l r-1$, as well as the enhanced responsiveness to light. This conclusion was corroborated through $b l r-1$ overexpression. The negative effect caused by blr-1 overexpression was reflected both in the activation and repression of light-regulated genes, and finally in photoconidiation, suggesting a global alteration in the perception and transduction of blue light by the alteration of the balance of the amounts of the BLR proteins. Although these results were unexpected and previously not described in fungi, they suggest that a delicate balance in BLR-1/BLR-2 levels is a major factor for the appropriate sensing and transduction of blue light. An alternative explanation is that overproduction of the $b l r-1$ transcript might trigger a strong negative regulation mechanism, affecting the efficiency of translation of the transcript. The effect of $b l r-1$ overexpression on light signal transduction is certainly interesting and deserves future indepth analysis.

It is noteworthy that, in addition to its regulation by red light (Rosales-Saavedra et al., 2006), we found that the expression of blu-4 is clearly regulated by blue light through the BLR complex. In agreement with this observation, analysis of the blu-4 promoter region in $T$. reesei indicated the presence of an LRE (GATTC- $\mathrm{N}_{21}$ CGATC) $725 \mathrm{bp}$ upstream of the putative ATG, as well as other GATA-type elements, as shown for other BLRdependent light-regulated genes (Rosales-Saavedra et al., 2006). To date, $b l u-4$ is the first gene that represents a point of convergence of the signal transduction cascades involved in the response to two different light inputs in fungi. Furthermore, the cross-talk between these two light perception systems might be, at least in part, responsible for the altered growth provoked by the overexpression of blr-2, probably due to an imbalance in the light regulation between the two (or more) inputs (Fig. 9a). In plants, cross-talk between cryptochromes and phytochromes that regulate plant growth and architecture has been well documented (Franklin et al., 2005).

In $N$. crassa, the expression of $w c$ genes is mainly regulated at the transcriptional level through WC proteins. This dependence on both WC proteins has been found both in the dark and after light induction (Ballario et al., 1996; Cheng et al., 2003; Káldi et al., 2006). In T. atroviride, the induction of blr-2 by light was faster and higher in the OEblr2-13 strain compared to the WT strain, similar to the expression pattern found for the blue-light-induced genes. We determined a $75 \%$ induction of $b l r-2$ in OEblr2 that represents a 9.2-fold increase with respect to the transcript levels found in the WT strain, strongly suggesting a positive post-transcriptional autoregulation. Analysis of the expression of $b l r-2$ in the $\Delta b l r-1$ mutant showed that its expression in the dark and accumulation induced by light depends on BLR-1, providing further support to the notion that the BLR proteins act as a complex in lightactivated post-transcriptional regulation (Fig. 9a). The fact that the transcript levels of $b l r-2$ decreased 3.5 -fold in the $\Delta b l r-1$ strain may be explained by the presence of a putative LRE (GATGC-N ${ }_{23}$-GATGC-N ${ }_{7}$-CGATT) $1122 \mathrm{nt}$ upstream of the ATG in the blr-2 promoter, similar to what is found in other light responsive genes, suggesting regulation at the transcriptional level mediated by the BLR complex in the dark (Fig. 9a). Consistently, the WC complex regulates the expression of $w c-1$ through two different promoters upstream of its ORF, named the distal and proximal promoters. The distal promoter is necessary for expression in the dark and the proximal promoter is essential for light-responsive expression (Káldi et al., 2006). Altogether, our data suggest that the BLR complex is a transcriptional regulator active even in the dark and that after a light pulse it can regulate expression of lightresponsive genes at the transcriptional level, and that of blr-2 at the post-transcriptional level (Fig. 9a).

Recently, we determined that the expression of lightregulated genes in $T$. atroviride is subjected to photoadaptation (Rosales-Saavedra et al., 2006). Similarly, blr-2 expression under constant illumination showed a typical adaptative response, similar to the expression pattern found after a light pulse, indicating the existence of a turnoff mechanism operating in its post-transcriptional regulation. Conversely, the expression pattern of the blr genes was different in the blr-2-overexpressing strain. However, the expression pattern of light-regulated genes was the same in the WT and OEblr2-13 strains, indicating that different adaptation mechanisms may be operating. In $N$. crassa, a negative feedback loop involving the flavoprotein VIVID and phosphorylation by different kinases that destabilize WC-1, and block the transcriptional activity of the WC complex, are the main mechanisms that control adaptation under continuous exposure to light (Schwerdtfeger \& Linden, 2003; He \& Liu, 2005). T. reesei, a close relative of T. atroviride, has a homologue (ENVOY) of VIVID that might play a similar role in photoadaptation (Schmoll et al., 2005). In our view, the mechanisms controlling the expression of $b l r-2$ at the post-transcriptional level and, possibly, mycelial growth under continuous exposure to light differ from those reported for $N$. crassa. The expression of blr-1 was not altered by light in the WT strain and an increase in the transcript levels was only evident in the $b l r-2$ overexpresser. The increase in the $b l r-1$ transcript steady-state levels observed only in a blr-2overexpressing strain was comparable to the change found in the blr-2 expression levels. The fluctuations observed in $b l r-1$ transcript levels have been observed in autoregulated genes, and typically arise from interference in the half-life 
of mRNA and the resulting time gap between transcription and translation, as shown for the zebrafish somitogenesis oscillator (Lewis, 2003; Schmoll et al., 2005). This may be indicative of the existence of a post-transcriptional regulation mechanism for both transcripts. However, since we did not find light-induced changes in the blr-1 mRNA steady-state levels in the WT strain, these results must await further experimental support.

Formation of complexes between the WC proteins (WCC) has been demonstrated (Cheng et al., 2002; Froehlich et al., 2002). The WC proteins have been found in the dark as a heterodimer able to bind LREs and, after a light pulse, a large WCC is formed that transiently binds the LREs of light-regulated genes in vivo (Froehlich et al., 2002; He \& Liu, 2005). Additionally, although WC-2 regulates WC-1 steady-state levels, WC-2 overexpression did not seem to alter the levels of WC-1 found in the WT strain (Cheng et al., 2001, 2002). Our data suggest that, similar to the WC proteins, the T. atroviride BLR proteins act as a complex. Additionally, blr-2 overexpression did not alter the transcript levels of blr-1 in the dark. Therefore, we consider that all phenotypes found may be associated directly with higher BLR-2 levels. Overexpression of blr-2 resulted in a threefold higher sensitivity to light of the blr-2-overexpressing strains than that of the WT strain. The fluence required for the light response was lower for the OEblr2 strains than for the WT strain. This parameter, directly associated with photoreceptors, was modified by blr-2 overexpression. The enhanced sensitivity to blue light of the OEblr2 strains can also explain the arrhythmic growth observed in the first light period, which possibly occurred as a result of an imbalance in the photosensitivity of different perception systems that regulate growth under constant illumination with white light. Our data strongly suggest that a BLR-1/BLR-2 complex is necessary for light perception. Although we cannot discard any effect associated with changes in the levels of BLR-1 caused by blr-2 overexpression, our data support the notion that a preformed photoreceptor complex between BLR proteins in the dark is key for the adequate perception and transduction of the light signal, as indicated in Fig. 9(b).

The BLR proteins regulate a set of specific genes, as demonstrated by the deletion of either of them (RosalesSaavedra et al., 2006), and the overexpression of blr-1 and blr-2. Together, these data clearly demonstrate a role dual for the BLR complex in light-dependent induction and repression of transcription (Fig. 9a). Here, we show evidence of additional roles of the BLR complex in the positive post-transcriptional regulation of blr-2 triggered by light, as well as of transcriptional regulation in the dark (Fig. 9a). The expression analysis of blr and early lightresponsive genes under constant illumination clearly indicates that a negative feedback loop is operating in the downregulation of the expression of these genes (Fig. 9a). Our results indicate that the adaptation mechanism to light is different between the blr and light-responsive genes, even though their regulation clearly occurs through the BLR complex. On the other hand, we found that the BLR proteins are dispensable for the regulation of growth of $T$. atroviride under defined photoperiods. However, there is strong evidence for cross-talk among the different light perception pathways. We demonstrated regulation of blu-4 expression by blue light through the BLR complex, in addition to its regulation by red light as described previously (Rosales-Saavedra et al., 2006), thus supporting cross-talk between the blue and red light transduction pathways. Finally, our results support a key role of BLR-2 in blue light sensing and transduction, and we propose that pre-formation of a BLR photoreceptor complex is key for appropriate light perception, and for subsequent robust regulation of all blue light responses (Fig. 9b).

\section{ACKNOWLEDGEMENTS}

This work was supported by grants CRP/MEX05-02 from the ICGEB Collaborative Research Programme and 47809-Q from Conacyt to A.H.-E. We are grateful to Dr Octavio Martínez for assistance with statistical analysis and to Dr John Délano. E. U.E.-N. is indebted to Conacyt, for a doctoral fellowship.

\section{REFERENCES}

Ambra, R., Grimaldi, B., Zamboni, S., Filetice, P., Macino, G. \& Ballario, P. (2004). Photomorphogenesis in the hypogeous fungus Tuber borchii: isolation and characterization of Tbwc-1, the homologue of the blue-light photoreceptor of Neurospora crassa. Fungal Genet Biol 41, 688-697.

Baek, J. M. \& Kenerley, C. M. (1998). The arg2 gene of Trichoderma virens: cloning and development of a homologous transformation system. Fungal Genet Biol 23, 34-44.

Ballario, P., Vittorioso, P., Magrelli, A., Talora, C., Cabibbo, A. \& Macino, G. (1996). White collar-1, a central regulator of blue light responses in Neurospora, is a zinc finger protein. EMBO J 15, 1650-1657.

Berrocal-Tito, G., Sametz-Baron, L., Eichenberg, K., Horwitz, B. A. \& Herrera-Estrella, A. (1999). Rapid blue light regulation of a Trichoderma harzianum photolyase gene. J Biol Chem 274, 14288-14294.

Berrocal-Tito, G. M., Rosales-Saavedra, M. T., Herrera-Estrella, A. \& Horwitz, B. A. (2000). Characterization of blue-light and developmental regulation of the photolyase gene phr-1 in Trichoderma harzianum. Photochem Photobiol 71, 662-668.

Casas-Flores, S., Rios-Momberg, M., Bibbins, M., Ponce-Noyola, P. \& Herrera-Estrella, A. (2004). BLR-1 and BLR-2, key regulatory elements of photoconidiation and mycelial grown in T. atroviride. Microbiology 150, 3561-3569.

Casas-Flores, S., Rios-Momberg, M., Rosales-Saavedra, T., Martínez-Hernández, P., Olmedo-Monfil, V. \& Herrera-Estrella, A. (2006). Cross talk between a fungal blue-light perception system and the cyclic AMP signaling pathway. Eukaryot Cell 5, 499-506.

Cheng, P., Yang, Y. \& Liu, Y. (2001). Interlocked feedback loops contribute to the robustness of the Neurospora circadian clock. Proc Natl Acad Sci U S A 98, 7408-7413.

Cheng, P., Yang, Y., Gardner, K. H. \& Liu, Y. (2002). PAS domainmediated WC-1/WC-2 interaction is essential for maintaining of steady-state level of WC-1 and of function of both proteins in circadian clock and light responses of Neurospora. Mol Cell Biol 22, $517-524$. 
Cheng, P., Yang, Y., Wang, L., He, Q. \& Liu, Y. (2003). WHITE COLLAR-1, a multifunctional Neurospora protein involved in the circadian feedback loops, light sensing and transcription repression of wc-2. J Biol Chem 278, 3801-3808.

Franchi, L., Fulci, V. \& Macino, G. (2005). Protein kinase C modulates light responses in Neurospora by regulating the blue light photoreceptor WC-1. Mol Microbiol 56, 334-345.

Franklin, K. A., Larner, V. S. \& Whitelam, G. C. (2005). The signal transducing photoreceptors of plants. Int J Dev Biol 49, 653-664.

Froehlich, A. C., Liu, Y., Loros, J. J. \& Dunlap, J. C. (2002). White collar-1, a circadian blue light photoreceptor, binding to the frequency promoter. Science 297, 815-819.

He, Q. \& Liu, Y. (2005). Molecular mechanism of light responses in Neurospora: from light-induced transcription to photoadaptation. Genes Dev 19, 2888-2899.

He, Q., Cheng, P., Yang, Y., Wang, L., Gardner, K. H. \& Liu, Y. (2002). White collar-1, a DNA binding transcription factor and a light sensor. Science 297, 840-843.

Herrera-Estrella, A., Goldman, G. H. \& Van Montagu, M. (1990). High efficiency transformation system for the biocontrol agents, Trichoderma spp. Mol Microbiol 4, 839-843.

Horwitz, B. A., Gressel, J. \& Malkin, S. (1985). Photoperception mutants in Trichoderma: mutants that sporulate in response to stress but not light. Curr Genet 9, 605-613.

Idnurm, A., Rodriguez-Romero, J., Carrochado, L. M., Sanz, C., Iturriaga, E. A., Eslava, A. P. \& Heitman, J. (2006). The Phycomyces madA gene encodes a blue-light photoreceptor for phototropism and other light responses. Proc Natl Acad Sci U S A 103, 4546-4551.

Jones, J. D., Dunsmuir, P. \& Bedbrook, J. (1985). High-level expression of induced chimeric genes in regenerated transformed plants. EMBO J 4, 2411-2418.

Káldi, K., Gonzalez, B. H. \& Brunner, M. (2006). Transcriptional regulation of the Neurospora circadian clock gene $w c-1$ affects the phase of circadian output. EMBO Rep 7, 199-204.

Lewis, J. (2003). Autoinhibition with transcriptional delay: a simple mechanism for the zebrafish somitogenesis oscillator. Curr Biol 13, 1398-1408.

Lewis, Z. A., Correa, A., Schwerdtfeger, C., Link, K. L., Xie, X., Gomer, R. H., Thomas, T., Ebbole, D. J. \& Bell-Pedersen, D. (2002). Overexpression of white collar-1 (WC-1) activates circadian clockassociated genes, but is not sufficient to induce most light-regulated gene expression in Neurospora crassa. Mol Microbiol 45, 917-931.

Linden, H. \& Macino, G. (1997). White collar 2, a partner in blue-light signal transduction, controlling expression of light-regulated genes in Neurospora crassa. EMBO J 16, 98-109.
Linden, H., Ballario, P. \& Macino, G. (1997). Blue light regulation in Neurospora crassa. Fungal Genet Biol 22, 141-150.

Liu, Y., He, Q. \& Cheng, P. (2003). Photoreception in Neurospora: a tale of two white collar proteins. Cell Mol Life Sci 60, 2131-2138.

Livak, K. J. \& Schmittgen, T. D. (2001). Analysis of relative gene expression data using real-time quantitative PCR and the $2^{-\Delta \Delta C_{\mathrm{T}}}$ method. Methods 25, 402-408.

Lu, Y. K., Sun, K. H. \& Shen, W. C. (2005). Blue light negatively regulates the sexual filamentation via the $\mathrm{Cwc1}$ and $\mathrm{Cwc} 2$ proteins in Cryptococcus neoformans. Mol Microbiol 56, 480-491.

Mach, R. L., Schindler, M. \& Kubicek, C. P. (1994). Transformation of Trichoderma reesei based on hygromycin B resistance using homologous expression signals. Curr Genet 25, 567-570.

Puyesky, M., Ponce-Noyola, P., Horwitz, B. A. \& Herrera-Estrella, A. (1997). Glyceraldehyde-3-phosphate dehydrogenase expression in Trichoderma harzianum is repressed during conidiation and mycoparasitism. Microbiology 143, 3157-3164.

Raeder, U. \& Broda, P. (1985). Rapid preparation of DNA from filamentous fungi. Lett Appl Microbiol 1, 17-20.

Rocha-Ramírez, V., Omero, C., Chet, I., Horwitz, B. A. \& HerreraEstrella, A. (2002). Trichoderma atroviride G-protein alpha-subunit gene tgal is involved in mycoparasitic coiling and conidiation. Eukaryot Cell 1, 594-605.

Rosales-Saavedra, T., Esquivel-Naranjo, E. U., Casas-Flores, S., Martínez-Hernández, P., Ibarra-Laclette, E., Cortes-Penagos, C. \& Herrera-Estrella, A. (2006). Novel light-regulated genes in $T$. atroviride: a dissection by cDNA microarrays. Microbiology 152, 3305-3317.

Sambrook, J., Fritsch, E. F. \& Maniatis, T. (1989). Molecular Cloning: a Laboratory Manual, 2nd edn. Cold Spring Harbor, NY: Cold Spring Harbor Laboratory.

Schmoll, M., Franchi, L. \& Kubicek, C. P. (2005). Envoy, a PAS/LOV domain protein of Hypocrea jecorina (anamorph Trichoderma reesei), modulates cellulase gene transcription in response to light. Eukaryot Cell 4, 1998-2007.

Schwerdtfeger, C. \& Linden, H. (2003). VIVID is a flavoprotein and serves as a fungal blue light photoreceptor for photoadaptation. EMBO J 22, 4846-4855.

Silva, F., Torres-Martínez, S. \& Garre, V. (2006). Distinct white collar1 genes control specific light responses in Mucor circinelloides. Mol Microbiol 61, 1023-1037.

Edited by: B. A. Horwitz 